\title{
Mucinosis: Treatment with Radiation Therapy A Case Report
}

\section{Jonathan Roubil | Kara Banson | James Shen | Kevin O'Connor | Kelly Fournier RT(T) | Andrea Hebert RT(T) | Raymond Geitler RT(T) | Thomas Quinn RT(T) | Carla Bradford | Nadine Bayless | Erica Smith RT(T) | Samantha Boudreau RT(T) | Allison Sacher | Jesse Aronowitz | Paul Rava | Maryann Bishop-Jodoin | Harry Bushe | Linda Ding | TJ FitzGerald*}

\section{*Correspondence: TJ FitzGerald}

Address: Department of Radiation Oncology, University of Massachusetts Medical School, 55 Lake Avenue North, Worcester MA 01655, USA

e-mail $\bowtie:$ TJ.FitzGerald@umassmemorial.org

Received: 30 April 2021; Accepted: 06 May 2021

Copyright: (C) 2021 Roubil J. This is an open-access article distributed under the terms of the Creative Commons Attribution License, which permits unrestricted use, distribution, and reproduction in any medium, provided that the original work is properly cited.

\section{ABSTRACT}

Mucin is a hyaluronic acid complex found in modest quantity in dermal connectives tissues. It serves as a protective barrier and supports tissue elasticity. Mucin has an important role as a response vehicle to injury and trauma in multiple body regions. Although thought to be produced by connective tissue, fibroblasts, and mast cells, the origin and regulatory mechanisms associated with production and absorption of mucin are largely unknown. Accumulation of unregulated large volumes of mucin in subcutaneous tissues can result in pain and limited wound healing when tissues over saturated with mucin are injured. Repair systems are less effective in edematous tissue as cell systems required for healing cannot accumulate at the site of injury in an organized enterprise manner. Clinically relevant forms of mucinosis have been described in hypothyroidism, thyrotoxicosis, and scleromyxedema associated with monoclonal gammopathies. Mucinosis has also been associated with systemic lupus, systemic sclerosis, and dermatomyositis including patients treated with chemoradiotherapy. In this paper we present a case report of an individual who had exhausted traditional therapies for mucinosis associated with an underlying thyroid disorder and had significant difficulty walking due to pain and discomfort in both her feet and distal lower extremities. We report on successful application of radiation therapy to effectively treat her symptoms. Keywords: Mucinosis, Radiation Therapy

\section{Introduction}

Mucinosis is a rare condition of exaggerated accumulation of hyaluronic acid complex in subcutaneous tissues (Biondo G et al., 2019). The deposits accumulate in any body region and can create considerable discomfort when deposited in areas of joint articulation, mucosal surfaces, and extremities. The condition can be primary when biopsies of skin reveal predominantly mucin or secondary when the mucin is associated with an additional dermatologic disease process such as dermatomyositis (Cohen $\mathrm{P}$ et al., 2020). Mucinosis has been described in patients after treatment of a primary malignancy. Although the mechanism is not well understood, it is postulated that rebound of post therapy cytokines stimulate 
glycosaminoglycan synthesis by fibroblasts promoting production of mucin in tissues including the skin. Reports suggest that Interleukin-1 and Interleukin-6 are elevated in patients with mucin deposition in dermal tissues with systemic lupus and dermatomyositis, however the findings must be considered nonspecific. Mucinosis is described in both hypothyroidism, hyperthyroidism, and scleromyxedema associated in patients with monoclonal gammopathies (Biondo et al., 2019; Cohen P et al., 2020).

Therapies have been limited and have included steroid therapy, thalidomide, and azathioprine. If mucinosis is generated as a secondary response to a primary disorder such as dermatomyositis, treating the underlying disorder can have a positive impact and influence outcome. Traditional therapies can have an impact, however often therapies have only modest impact and at best, only partially effective. Cases resistant to therapy have been treated with intravenous immunoglobulin (IVIG) (Dolenc-Voljc M et al., 2013).

Radiation therapy remains an effective method of therapy for patients with non-malignant proliferative disorders including heterotopic bone formation and keloids. In this case report, we discuss a patient with longstanding mucinosis associated with a previous thyroid disorder with considerable limitation in ambulation due to mucin deposition in her feet requiring her to walk with support. She has exhausted more traditional therapies and our service was consulted to discuss radiation therapy as an approach to her care. One of the authors (TJF) had experience in treating patients with amyotrophic lateral sclerosis and patients with neurodegenerative disorders to limit salivary function and has treated an additional patient with significant thermal injury with a non-healing salivary-cutaneous fistula which successfully closed after radiation therapy. Therefore, similar approach to dose volume application was applied to this situation (Favarato et al., 2013; Harris JE et al., 2004; Kuo KL et al., 2017; Rongioletti F, 2006; Rongioletti F and Rebora A, 2001a; Rongioletti F and Rebora A, 2001b; Singh S, et al., 2013).

\section{Case Presentation}

The patient is a 52-year-old female who had a longstanding history of thyrotoxicosis treated with radioactive iodine with resultant hypothyroidism. Of more than 30 medical co-morbidities, pertinent relationships included peripheral vascular disease and lupus anticoagulant with hypercoagulable state. The patient listed 19 allergies. She underwent many surgical interventions and topical therapies with limited to no success. Pathology from surgical procedures confirmed superficial and deep mucin deposition with underlying dense nodular and hypocellular collagenous proliferation. In 2003, she was initially seen by our service to evaluate the role of radiation therapy to multiple subcutaneous nodular regions of mucinosis as she had limited to no response to other therapies. She received 3000 cGy in 200 cGy fractions to two sites of involvement using intensity modulation to create a circular near- 
circumferential target dose distribution. We were recently asked to re-visit with the patient to evaluate her for significant progressive changes and deposition of mucin involving both feet including the dorsal surface of her toes. Her clinical situation had deteriorated, and she was orthopedically challenged secondary to mucinosis. She required support while walking. Her shoes could not properly fit her feet and she was progressively getting worse. Her medical situation required subspecialty support from more than eight subspecialty services including dermatology, endocrinology, and vascular surgery.

At the time of consultation patient had limited ambulation and significant pain unresolved with conservative measures including limited physical therapy and leg elevation. Treatment was offered and the patient agreed as she felt alternative therapy was exhausted. Multiple sites at/around the foot and ankle were treated using $9 \mathrm{MeV}$ electrons with bolus to treat the areas of concern. 3000 cGy were delivered in 200 cGy fractions calculated to the $90 \%$ isodose line with blocking used at the level of the growth plates of the toenails. Planning CT demonstrating the target are is seen in Fig. 1 and the visual target in the feet is seen in Fig. 2.

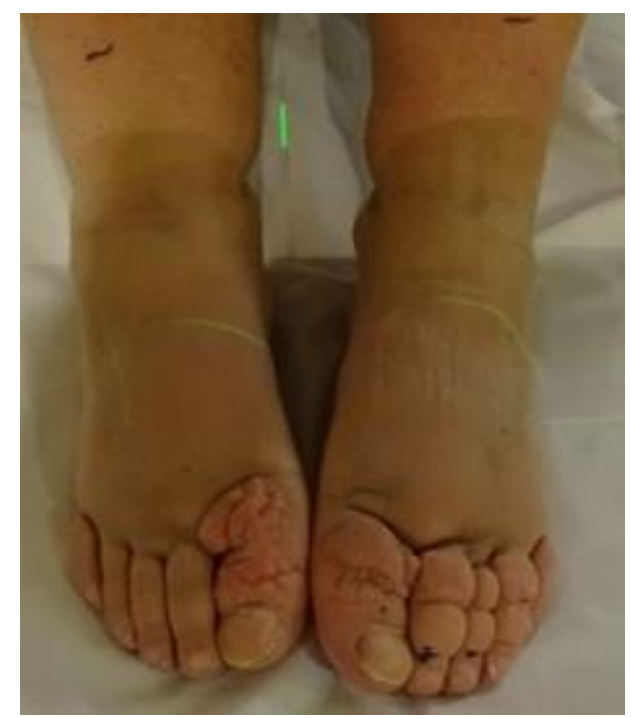

Figure1: Pre-therapy image of the dorsal surface of both right and left foot.
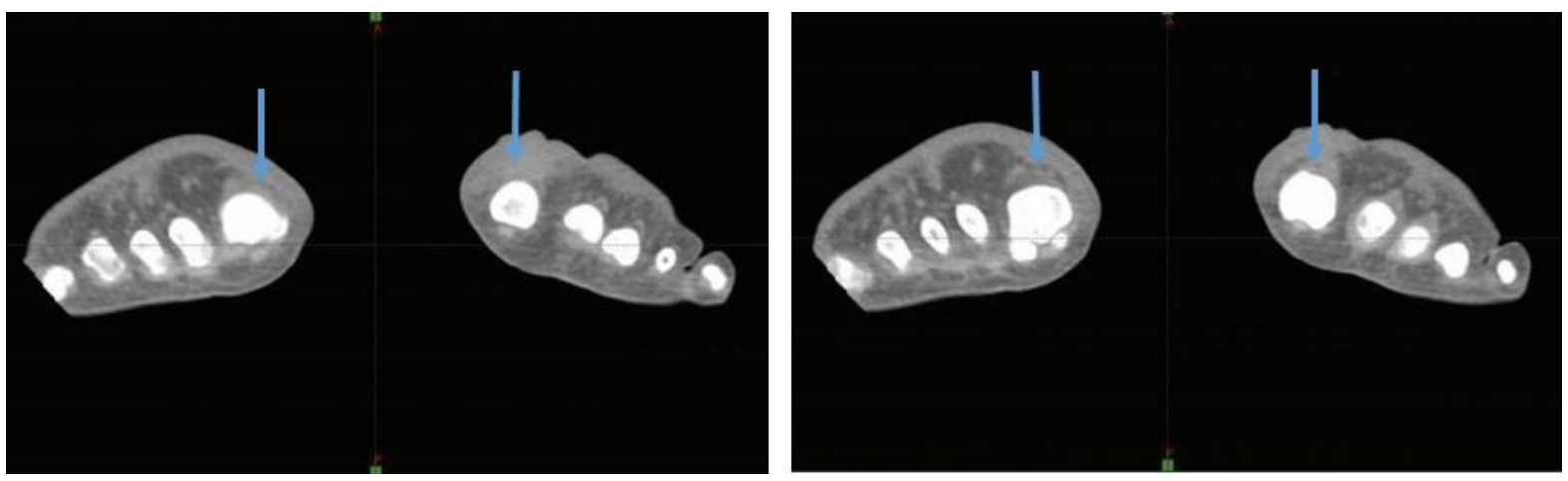

Figure 2: Planning computer tomography demonstrating mucin accumulation in soft tissues. 


\section{Clinical Outcome}

The patient tolerated the course of therapy relatively well. There is nuance to treating multiple sloped surfaces with both electrons and photons and the therapy was imposed onto surfaces that were uniformly abnormal pre-therapy with associated changes consistent with myxedema and interstitial fibrosis. Bolus was applied to the growth area of the toenails to intentional titrate dose to the growth site. Post therapy, the patient had moist desquamation of the sloped surfaces of the right and left foot ankle which could not be accommodated through planning with electron therapy as we had done years earlier with intensity modulated photon therapy. Sloped and non-uniform surfaces coupled with interstitial edema and mucin deposition retarded healing from relatively low dose therapy. Once treated, she did require medical pain medication augmentation for short term pain control. Once pain management was established and maintained, physical and rehabilitation therapy was able to be initiated and with constant attention, the patient demonstrated significant improvement. She is now one year removed from therapy and she is walking without support and driving her own car which she could not easily achieve pre-therapy.

\section{Discussion}

Radiation oncologists have experience in treating surfaces of the foot in many disease settings including Kaposi sarcoma, mycosis fungoides, and pediatric rhabdomyosarcoma (Jyothirmayi et al., 1999). Treatment of the foot requires careful planning to surfaces sensitive to therapy including the sole of the foot and the multiple sloped surfaces along the medial and lateral surfaces. Advanced technology planning often cannot accommodate every facet of topography of the foot and oncologists need to prioritize goals and objectives with compromise of objectives which cannot be met. In the ideal setting, partial volume therapy lends itself to less sequelae of management. In this case, however, partial volume therapy to a limited area of disease would have permitted reaccumulating mucin from other regions, therefore the entire structure and dorsal surface foot required therapy to achieve the desired outcome. In diseases that involve the topical surfaces of the majority of the foot such as mycosis and mucinosis, sequelae may occur as the tissue itself is compromised from the disease process and often can not heal in the more timely manner seen when the skin is an unintentional target of treatment as seen in this patient. Likewise, dermal stem cells are challenged migrating into areas treated when compromised by disease and myxedema, therefore healing of injury can be protracted and requires time and thoughtful management (Jyothirmayi et al., 1999).

The dose, fractionation, and volume chosen for therapy are important considerations. The hyaluronic acid and mucin are thought to be generated from connective tissue; therefore the target of 
therapy is likely the dermis of the skin. The objective is to titrate the production of mucin from these tissues. Although an imperfect correlation, radiation doses of greater than 3000 cGy are known to titrate secretions from the parotid gland and one author (TJF) has experience treating a burn injury patient with a parotid cutaneous fistula which closed and healed after receiving 3000 cGy. In this patient 3000 cGy was effective in 2003 and was again effective in 2020, however the multiple sloped surfaces and preexisting dermal and interstitial edema required attention to detail to achieve a good outcome.

Physical and rehabilitation therapy is an essential component to care for all patients treated with surgery and/or radiation therapy to the extremity. In this patient, optimal outcome could not have been achieved without these important services. Extremity patients, including this patient, present with compromised tissues and limited vascularity. Healing is delayed because of mucin deposition de novo, therefore mobilization of mucin with vigorous post therapy care is important for optimal outcome. While radiation therapy abated the stimulus for form mucin in the connective tissue, compression and physical therapy post radiation were the mechanism to establish a durable and meaningful outcome for the patient. Similar to rehabilitation therapy for patients treated to extremities for definitive oncology management, comparable patients require a comprehensive approach to rehabilitation for optimal longterm functional outcome. This needs to be built into a survivorship model for patients post treatment.

Radiation therapy has been used for more nearly a century for diseases of non-malignant origin and remains important for several diseases today including heterotopic bone and keloid formation. In this paper we present a patient with mucinosis generated by an established thyroid disorder who had exhausted therapies for her situation. Radiation therapy remains an option for care if more traditional therapies prove less effective (Cohen PR et al., 2020; Harris JE et al., 2004; Kuo KL et al., 2017; Rongioletti F and Rebora A, 2001b).

\section{Conclusion}

Radiation therapy remains an important component of care for the patient with malignant and nonmalignant disease in the extremity. Mucinosis is a rare condition, however, can be malevolent in its behavior and have significant impact on the well being of patients. This patient had significant pain and discomfort secondary to mucin deposition in her feet and had exhausted traditional therapy including rehabilitation and physical therapy. Radiationfig therapy was applied to limit mucin production in the area of discomfort and with post therapy rehabilitation a good functional outcome was achieved in this unique clinical situation. 


\section{References}

Apisarnthanarax N, Ha CS, Duvic M. Mycosis fungoides with follicular mucinosis displaying aggressive tumor-stage transformation: successful treatment using radiation therapy plus oral bexarotene combination therapy. American Journal of Clinical Dermatology 2003; 4: 429-433.

Biondo G, Sola S, Pastorino C, Massone C. Clinical, dermoscopic, and histologic aspects of two cases of cutaneous focal mucinosis. Anais Brasileiros de Dermatologia 2019; 94: 334-336.

Bonta MD, Tannous ZS, Demierre MF, Gonzalez E, Harris NL, Duncan LM. Rapidly progressing mycosis fungoides presenting as follicular mucinosis. Journal of the American Academy of Dermatology 2000; 43: 635-640.

Cohen PR, Erickson CP, Calame A. Case report and review of solitary cutaneous focal mucinosis: a unique primary cutaneous mucinosis unrelated to mucinosis- associated systemic diseases. Dermatology Online Journal 2020; 26(8).

Dolenc-Voljč M, Jurčić V, Hočevar A, Tomšič M. Scleromyxedema with subcutaneous nodules: successful treatment with thalidomide and intravenous immunoglobulin. Case Reports in Dermatology 2013; 5: 309-315.

Favarato MH, Miranda SS, Caleiro MT, Assad AP, Halpern I, Fuller R. Cutaneous mucinosis in mixed connective tissue disease. Anais Brasileiros de Dermatologia 2013; 88: 635-638.

Harris JE, Purcell SM, Griffin TD. Acral persistent papular mucinosis. Journal of the American Academy of Dermatology 2004; 51: 982-988.

Jyothirmayi R, Sittampalam Y, Harmer C. Soft tissue sarcoma of the hand and foot: conservative surgery and radiation therapy. Sarcoma 1999; 3: 17-24.

Kuo KL, Lee LY, Kuo TT. Solitary cutaneous focal mucinosis: A clinicopathological study of 11 cases of soft fibroma-like cutaneous mucinous lesions. The Journal of Dermatology 2017; 44: 335-338.

Rongioletti F and Rebora A. Cutaneous mucinoses: microscopic criteria for diagnosis. The American Journal of Dermatopathology 2001b; 23: 257-267.

Rongioletti F and Rebora A. Updated classification of papular mucinosis, lichen myxedematosus, and scleromyxedema. Journal of the American Academy of Dermatology 2001a; 44: 273-281.

Rongioletti F. Lichen myxedematosus (papular mucinosis): new concepts and perspectives for an old disease. Seminars in Cutaneous Medicine and Surgery 2006; 25: 100-104.

Singh S, Kanwar AJ, Saikia UN. Scleromyxoedema with disseminated subcutaneous nodules: rare presentation of an uncommon dermatosis. Clinical and Experimental Dermatology 2013; 38: 36-39. 